Confinement-induced phase transition in a DNA-lipid hydrated complex

This article has been downloaded from IOPscience. Please scroll down to see the full text article.

2010 EPL 9128001

(http://iopscience.iop.org/0295-5075/91/2/28001)

View the table of contents for this issue, or go to the journal homepage for more

Download details:

IP Address: 147.210.83.198

The article was downloaded on 20/10/2010 at 09:26

Please note that terms and conditions apply. 


\title{
Confinement-induced phase transition in a DNA-lipid hydrated complex
}

\author{
E. Andreoli de Oliveira ${ }^{1}$, E. R. Teixeira da Silva ${ }^{1,2}$, A. FÉvrier ${ }^{2}$, É. Grelet $^{2}$, F. Nallet $^{2}$ \\ and L. NAVAILLES ${ }^{2}$ \\ ${ }^{1}$ Instituto de Física, Universidade de São Paulo - Rua do Matão Travessa R Nr. 187, Brasil \\ ${ }^{2}$ Université de Bordeaux, Centre de recherche Paul-Pascal, CNRS - 115 avenue du Docteur-Schweitzer, \\ F-33600 Pessac, France, EU
}

received 29 April 2010; accepted in final form 7 July 2010

published online 4 August 2010

PACS 82.70.-y - Disperse systems; complex fluids

PACS 68.18.Jk - Phase transitions in liquid thin films

PACS 87.15.-v-Biomolecules: structure and physical properties

\begin{abstract}
We study the effect of the soft confinement by fluid lipid bilayers on the spatial organisation of DNA molecules in a DNA-zwitterionic lipid hydrated lamellar complex. The confinement is increased by dehydrating the complex in a controlled way, which leads to a decrease of the water channel thickness separating the periodically stacked bilayers. Using grazing-incidence small-angle X-ray scattering on an oriented thin film, we probe in situ as dehydration proceeds the structure of the DNA-lipid complex. A structural phase transition is evidenced, where an apparently disordered phase of DNA rods embedded within the one-dimensionally ordered lipid lamellar phase observed at high hydration is replaced by a $2 \mathrm{D}$ hexagonal structure of DNA molecules intercalated between the lipid bilayers.
\end{abstract}

Copyright (C) EPLA, 2010

Introduction. - The design of new materials based on the manipulation of molecular or atomic ordering has stimulated the investigation on confinement effects, both experimentally [1-6] and theoretically [7-10]. At molecular level, the confinement of a simple fluid induces ordering and also a reduction in the number of nearest-neighbour molecules, leading to changes in the phase behaviour of the fluid. Computer simulations or phenomenological models have been applied to study simple confined fluids however, from an experimental point of view, soft materials such as colloids and mixtures of polymers are more attractive model systems $[11,12]$ since the strength and the range of effective interactions can be varied over a wide range.

The lamellar phase, formed by regularly stacked surfactant bilayers separated by solvent layers, has been used as a template to prepare composite materials, by inserting colloidal particles or polymers into the solvent (usually water) layers [13-22]. A soft confinement can be imposed to the particles, either by changing the bilayer rigidity or the bilayer separation, allowing the control of physical properties of the composite. Interactions of biomolecules and lamellar phases formed by lipids have been intensively studied in the last years as model systems to investigate many biological processes $[23,24]$. In this context, complexes formed by lipids and DNA, preferably concentrated ones in view of transfection applications, have been actively studied, revealing a rich polymorphism and a variety of structures [17,25-34]. Complexation of DNA with lipids can be mediated by electrostatic interactions - usually, either by divalent cations $[26,28,30,33]$ or by adding cationic lipids to the membrane $[17,25,31,32,34]$. As proposed theoretically [35], complexation could also be mediated by an entropic mechanism, and complexes formed without the aid of divalent cations or cationic lipids have indeed been experimentally reported at moderate concentrations $[27,29]$.

Double-stranded DNA is a semiflexible polyelectrolyte that can be intercalated between the lipid bilayers in different spatial organisations, depending on composition of the membrane and temperature, including a 2D analogue of the nematic phase - with orientational in-plane orderand a columnar mesophase - with out-of-plane positional ordering [29,36-38]. The transition from a $2 \mathrm{D}$ nematic to a columnar phase has been observed in different systems, related to a transition in the lipid organisation from a fluid $L_{\alpha}$ lamellar phase to a gel $L_{\beta}$ phase $[28,31,34,37]$.

We investigate the effect of the soft confinement by fluid lipid bilayers on the spatial organisation of DNA molecules in a DNA-zwitterionic lipid complex in water. The confinement is increased by decreasing the water 
content in a controlled way, which amounts to reducing the separation between adjacent bilayers. Using grazingincidence small-angle X-ray scattering on an oriented thin supported film, we follow in situ the structure as dehydration proceeds. A structural phase transition is evidenced, where the high-dilution, disordered or possibly 2D nematic, phase of DNA rods loosely confined by the lipid bilayers is replaced by a significantly more concentrated 2D hexagonal structure of DNA molecules intercalated between lipid bilayers, themselves still forming a fluid $L_{\alpha}$ lamellar phase.

Materials and methods. - The host system for DNA is prepared by adding pure water to a mixture of a zwitterionic lipid (soya lecithin-phosphatidylcholine headgroup or PC) and a non-ionic co-surfactant (simulsol), with the $\mathrm{PC} /$ simulsol mass ratio kept constant and equal to $7 / 3$. As evidenced by polarised light microscopy and smallangle X-ray scattering, a lamellar structure of periodically stacked bilayers is obtained at room temperature up to the dilution limit (water volume fraction) $\phi_{w}=0.60$. While varying the water content from $\phi_{w}=0.25$ to the dilution limit, the period of the lamellar stack $(D)$ increases from $4.8 \mathrm{~nm}$ to $9.2 \mathrm{~nm}$, and follows the usual one-dimensional swelling law $D=\delta /\left(1-\phi_{w}\right)$. This leads to a bilayer thickness $\delta$ of ca. $3.6 \mathrm{~nm}$. An estimate for the height of the water channels is therefore given by $\delta \phi_{w} /\left(1-\phi_{w}\right)$.

DNA fragments prepared by sonicating calf thymus DNA are then added to the host lamellar phase. The ultra-sound treatment (followed by the intercalation of the fluorescent dye Y3601-Invitrogen, Carlsbad, USA) leads to DNA pieces of ca. 150 base pairs, or a contour length $L$ around $50 \mathrm{~nm}$, i.e. the DNA persistence length: the DNA molecules are thus appropriately described as rods, with a diameter taken to be $2.4 \mathrm{~nm}$. In the present study, the initial sample composition (volume fraction) is chosen to be $\phi_{w}=0.319, \phi_{\mathrm{DNA}}=0.074$ and $\phi_{\text {lip }}=0.607$, the latter value lumping together the lipid and co-surfactant with $\mathrm{PC} /$ simulsol $=7 / 3$. Upon hydrating or dehydrating the sample ("dilution line"), the lipid-to-DNA volume ratio, namely $\rho=8.2$, keeps a constant value as long as the sample remains a single phase. After a thorough mixing of the components, the sample is left to settle at room temperature for 2 months. Visual inspection suggests a macroscopically homogeneous sample, which is confirmed by polarised light (fig. 1a) and fluorescence (fig. 1b) microscopy observations. As evidenced by the typical texture displayed in fig. 1a, the sample is a lamellar phase.

In order to vary the hydration state of the DNAlipid complex, we are using a home-made humidity and temperature control device specially designed for grazingincidence small-angle X-ray scattering. Our device is derived from the design described by Pieranski [39], with a sample stage at temperature $T_{s}=35^{\circ} \mathrm{C}$ above a water reservoir at a variable temperature $T_{w}$, both enclosed in an air-tight chamber. Two windows covered with Kapton foils allow the incident, reflected and scattered

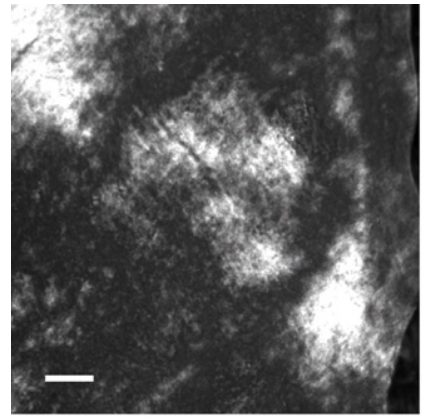

(a)

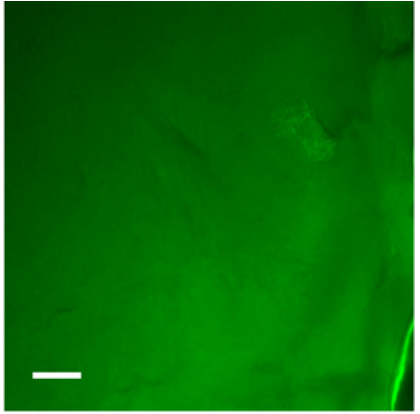

(b)
Fig. 1: (Colour on-line) Polarised light a), and fluorescence b) microscopy pictures of the initial state namely, a homogeneous lamellar phase, of the DNA-lipid complex in water $-\phi_{w}=$ $0.319, \phi_{\text {lip }}=0.607, \phi_{\text {DNA }}=0.074$. Scale bar: $10 \mu \mathrm{m}$.

beams to enter or exit the device. When $T_{w}$ is set equal to $T_{s}$, a $100 \%$ relative-humidity state is reached at equilibrium in the chamber atmosphere. The water temperature may then be set to a value below $T_{s}$, which results in sample dehydration at a rate depending on (and actually increasing with) the difference $T_{s}-T_{w}$.

For the X-ray scattering experiment, the sample is deposited onto a standard glass slide (thickness of about $1 \mathrm{~mm}$ ) as used for optical microscopy and, in order to form a thin film, manually scraped against it with another glass slide submitted to a gentle pressure. The procedure does of course not allow to precisely tune the film thickness: it may typically range from a few micrometres to a few ten micrometres, with observable variations across the ca. $1 \mathrm{~cm}^{2}$ film area. Once prepared, the supported film is rapidly inserted into the humidity chamber with temperature controls set for a $100 \%$ relative humidity. Owing to the above-described experimental constraints, the hydration state of the DNA-lipid complex immediately after film preparation is not precisely known, unfortunately. However, when the film arrives at equilibrium in the humidity chamber, the dilution limit is reached.

The beam line geometry (GMT instrument, BM32 at ESRF, Grenoble, France) is the one appropriate for grazing-incidence small-angle X-ray scattering. The incidence of the beam with respect to the nearly horizontal film is controlled by a goniometer with its horizontal axis $x$ perpendicular to the incident X-rays. The specular reflection deflects the beam by an angle $2 \theta$. The projection of the-nearly vertical-stacking axis in the detector plane defines axis $z$. The azimuthal coordinate in the detector plane is $\chi$ (see footnote $\left.{ }^{1}\right)$.

\footnotetext{
${ }^{1}$ The detector we use is a thermoelectrically cooled CCD camera (Princeton instrument, $1242 \times 1152$ pixels), borne by a goniometer moving in the vertical plane, $1146 \mathrm{~mm}$ from the sample. The wavelength of the monochromatic beam is set to $\lambda=0.103 \mathrm{~nm}$. The beam imprint onto the film is defined by slits $(0.5 \mathrm{~mm}$ horizontal, $0.025 \mathrm{~mm}$ vertical). It varies with the incidence of the X-ray beam.
} 

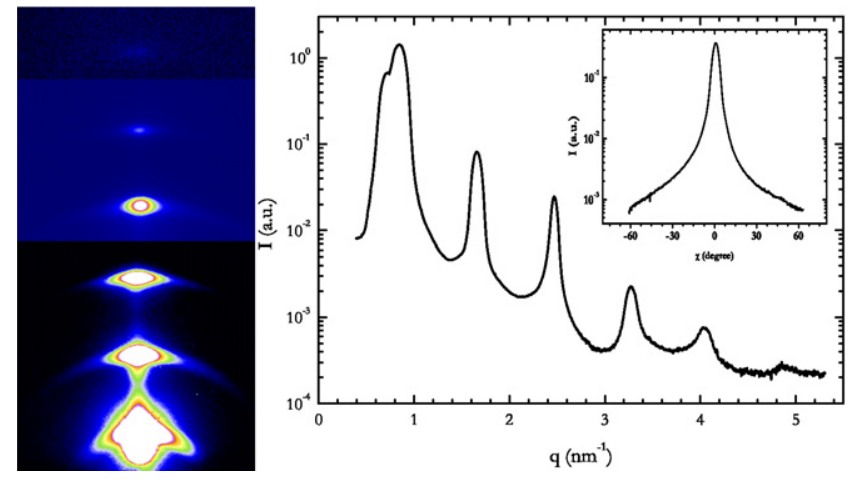

Fig. 2: (Colour on-line) Left: merged spectra obtained for three distinct grazing incidences on a homeotropically oriented lamellar DNA-lipid complex at high hydration, with Bragg reflections along axis $z$. Reciprocal space spanned: $0.5 \leqslant q_{z} \leqslant$ $5.4 \mathrm{~nm}^{-1}$ and $-1.2 \leqslant q_{x} \leqslant 1.2 \mathrm{~nm}^{-1}$. Right: azimuthally averaged scattered intensity as a function of the wave vector modulus $q$, exhibiting 6 Bragg peaks - the hump on the left of the first-order Bragg peak comes from the specular reflection. Inset: variation of the azimuthal intensity, radially averaged along $\chi$ in a thin corona centred around the first-order Bragg position.

Figure 2 displays the scattering data taken while the film is being hydrated in the humidity chamber. This snapshot indicates that the supported film is fairly well oriented with the stacking axis of the lamellae normal to the glass slide (homeotropic orientation) since the diffracted intensity appears as regularly spaced Bragg spots localised along the vertical axis $z$. The azimuthally averaged scattered intensity is displayed as a function of the modulus $q$ of the scattering wave vector in the right part of fig. 2, with up to 6 diffraction orders in the ratio $1: 2: 3: 4: 5: 6$ as expected for a one-dimensional periodic structure. The inset in the same fig. 2 corresponds to the radially averaged intensity at the position of the first-order Bragg peak: the narrow width of the line (FWHM $5.4 \mathrm{deg}$ ) confirms that the orientation of the film is indeed quite good.

While the film is being hydrated in the humidity chamber set for a $100 \%$ relative humidity, a few spectra are recorded to follow quantitatively the swelling of the structure. The lamellar stacking period $D$ as a function of time is displayed in fig. 3 . The sample continuously swells in ca. 3 hours to reach $D=8.95 \mathrm{~nm}$ (asymptotic fitted value). The swelling limit expected for a DNA-free lamellar phase with the same bilayer composition (for bulk samples in capillaries) is $D=9.2 \mathrm{~nm}$, implying that the sample is very close - but not yet at - full hydration.

Results. - The main results to be discussed here are obtained as the settings of the humidity control device are changed in order to start slowly dehydrating the fully swollen sample ${ }^{2}$.

\footnotetext{
${ }^{2}$ The total duration of the dehydration step being about 30 hours, much longer than the acquisition time (1-10 s) of a given spectrum, we assume that each spectrum is characteristic of the instantaneous (quasi) equilibrium structure of the DNA-lipid hydrated complex.
}

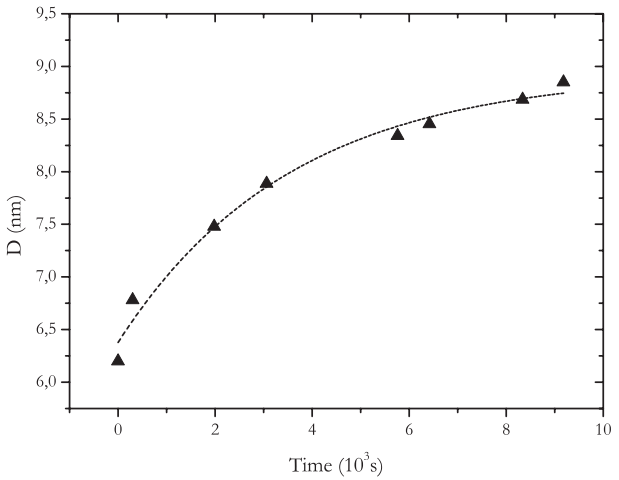

Fig. 3: Smectic period $D$ of the lamellar phase as a function of time. The DNA-lipid film continuously swells up to $8.9 \mathrm{~nm}$, i.e. close to the swelling limit expected for the DNA-free lamellar phase. The line corresponds to a single-exponential relaxation, with characteristic time $\tau=3.57 \times 10^{3} \mathrm{~s}$.

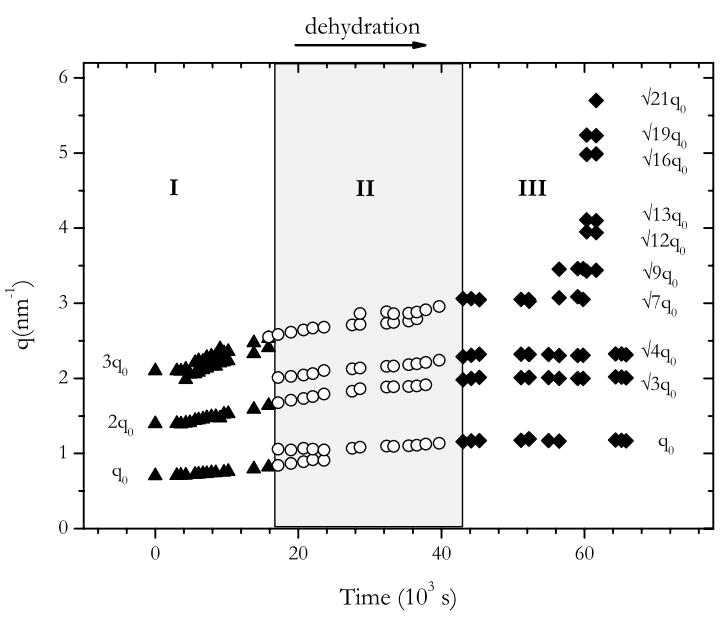

Fig. 4: Wave vector values of the Bragg peaks as a function of time while the DNA-lipid film is being continuously dehydrated. The two vertical lines mark the boundaries between three domains, see text for details.

The plot in fig. 4 displays as a function of time the locations in reciprocal space of all the peaks (or significant shoulders) observed in azimuthally averaged spectra, i.e. irrespective of their on-axis (along $z$ ) or off-axis position. Three distinct domains may be identified from the data, with boundaries reported as two vertical lines in fig. 4 .

In domain I, there is no off-axis scattering observable in the experimentally accessible range of reciprocal space. The spectra, very similar to the one displayed in fig. 2, have a clear one-dimensional character and are broadly consistent with a simple, gradually more confined lamellar structure. The stacking period decreases from $D=8.95 \mathrm{~nm}$ for the more hydrated sample to $7.67 \mathrm{~nm}$ at the boundary with the next domain.

In domain III, the observed Bragg spots (including a $\sqrt{21}$ peak, clearly distinct in the long-time limit, see fig. 5) are easily indexed as corresponding to a 2D hexagonal structure, with a lattice parameter first significantly 

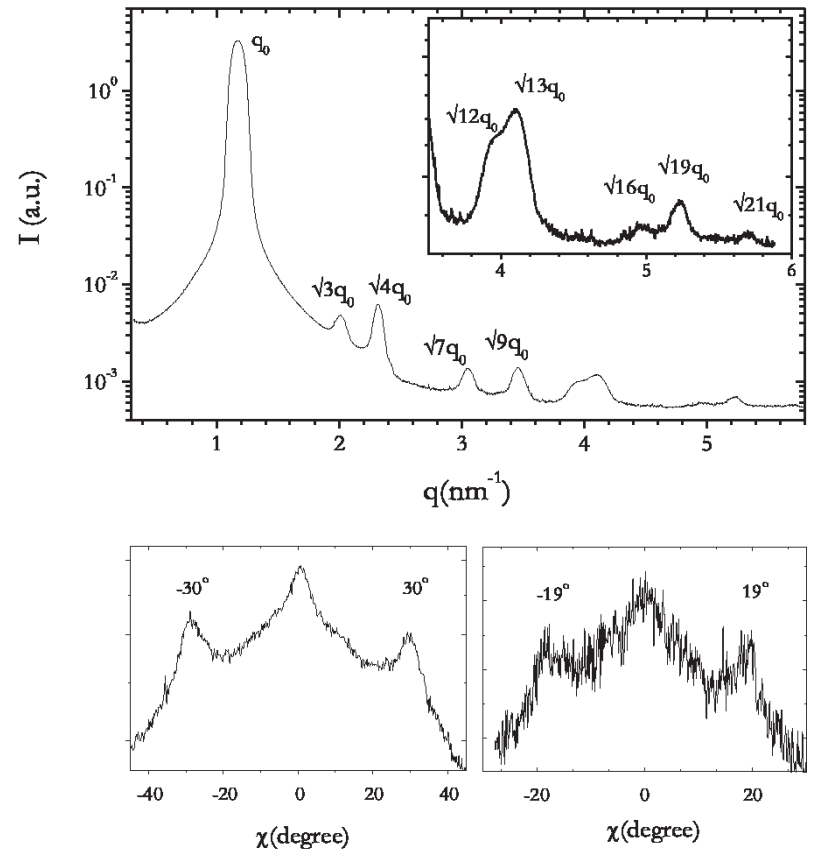

Fig. 5: Top: azimuthally averaged spectrum, characteristic of the later stage of domain III, where a 2D hexagonal structure is found. Bottom: radially averaged spectra, at the two positions $\sqrt{3} q_{0}$ and $\sqrt{7} q_{0}$, exhibiting the expected off-axis peaks at $\chi= \pm 30 \mathrm{deg}$ and $\chi= \pm 19 \mathrm{deg}$, respectively.

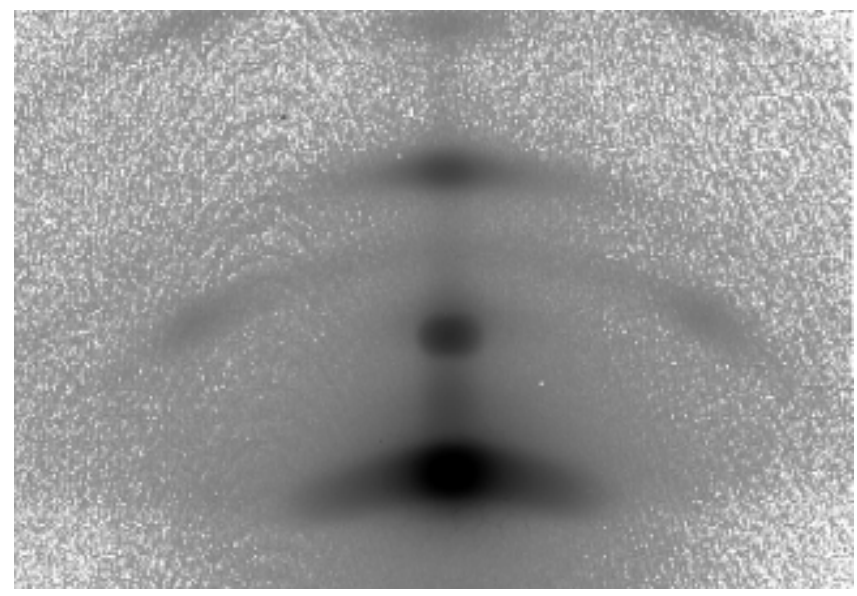

Fig. 6: X-ray scattering pattern representative of the complex spectra observed in the later part of domain II. The DNA-lipid film remains oriented with respect to the beam line axes and exhibits well defined off-axis diffraction peaks.

decreasing as time proceeds, nearly constant later and equal to $a=6.18 \mathrm{~nm}$.

Figure 5 also indicates that the off-axis signal, when observable, is nicely consistent with the $2 \mathrm{D}$ hexagonal structural identification. The supported film thus remains oriented across the transition form the 1D lamellar to the 2D columnar mesophase.

The intermediate domain II is not so easily described, however, as many distinct peaks and shoulders without (a)

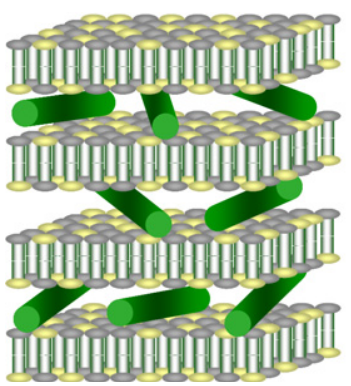

(b)

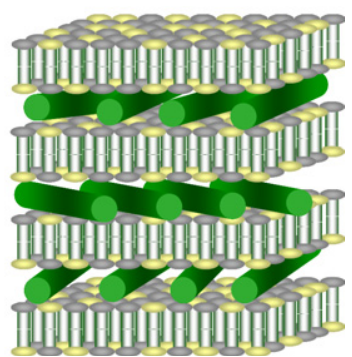

Fig. 7: (Colour on-line) Schematic representations of a) 2D isotropic DNA order inside the lamellar phase, or b) $2 \mathrm{D}$ nematic DNA order.

an obvious indexation are observed in the azimuthallyaveraged spectra. No off-axis signal is observable in the earlier part of domain II but, as displayed in fig. 6, offaxis Bragg spots clearly appear at later stages. Some of the peaks may be assigned to a lamellar structure in the earlier part of domain II and, similarly for the later part, other peaks are assigned to a 2D hexagonal structure. We have not found a wholly consistent description of the observed spectra in terms of a simple coexistence of the two abovementioned structures as the limited quality of the film orientation prevents us from assigning unambiguously offaxis peaks in the central part of domain II.

Interpretation. - The observed spectra in domain I are compatible with two distinct types of order for the DNA rods embedded in a lamellar host phase, either a $2 \mathrm{D}$ nematic or an isotropic phase, see fig. 7 for a schematic representation of these structural models.

In both models, the long axes of the DNA rods are perpendicular to the stacking axis of the bilayers owing to confinement - the water channel height is much smaller than the rod length. DNA axes remain isotropically distributed in planes parallel to the average bilayer plane for the (2D) isotropic model (fig. 7a), whereas they are on average parallel to a common axis in the $2 \mathrm{D}$ nematic model (fig. 7b). Note however that the direction of the common axis is not maintained along the lamellar stacking axis. These structural differences lead in principle to different scattering features: in the grazing incidence geometry, with the lamellae nearly parallel to the incident beam, the positional correlations between nearby DNA rods within a given water channel are expected to give rise to intensity maxima in the horizontal plane, roughly located at the reciprocal of the mean distance between adjacent DNA molecules. In the 2D isotropic model, where the water-DNA solution has to be dilute, the correlations are weak and the mean distance large. In the $2 \mathrm{D}$ nematic model, and only for such water channels that happen to have the nematic director parallel to the incident beam, correlations are much stronger and the mean distance is smaller. In any case, however, the reciprocal space 


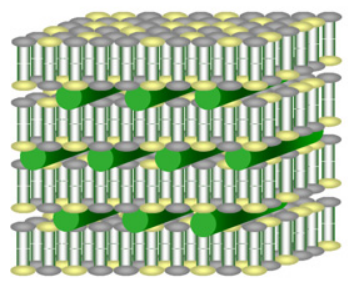

(a)

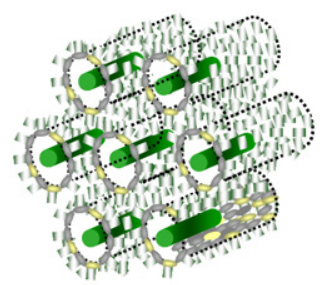

(b)
Fig. 8: (Colour on-line) Schematic representations of a) 2D hexagonal DNA assembly within the lipid lamellar phase, or b) inverted hexagonal lipid phase, with water cylinders coaxial to DNA molecules.

region where the scattering signature of the correlations is expected to be found - namely, at or close to the horizontal axis - is not experimentally accessible because it is "below the horizon", in other words shadowed by the (slightly) tilted glass slide supporting the oriented film. Therefore, there is no direct way here to discriminate between the two structural models. That is the reason why we prepared another sample with $\rho=8.4$ similar to the initial sample $(\rho=8.2)$, adjusting the water content to reach a stacking period $D$ of $7.61 \mathrm{~nm}$, i.e. close to the values observed near the end of domain I $(D=7.67 \mathrm{~nm})$. The powder X-ray scattering pattern recorded with a standard small-angle camera (data not shown) does not yield the characteristic features of nematic correlations within the water channels [29].

The spectra in domain III, characteristic of a 2D hexagonal symmetry, may correspond to different organisations of DNA and lipid components at the molecular level, with either a $2 \mathrm{D}$ hexagonal assembly of the DNA rods embedded within the lamellar structure of the lipid bilayer (fig. 8a), or an inverted hexagonal phase of lipids with the DNA rods fitting into the water cylindrical channels (fig. 8b).

As far as the Bragg peak locations are concerned, the scattering features of the two models are identical. Only minute differences are expected in diffuse scattering, originating in the form factor differences between a water cylinder and a water platelet. In order to nevertheless discriminate between the two models, we take advantage of their large texture differences - oily streak vs. fanshape textures [40] - usually observed in polarising optical microscopy. We prepared with the same initial sample another supported thin film left dehydrating in the humidity control device. The picture displayed in fig. 9, with an oily streak texture typical for a lamellar phase, definitely supports our interpretation that the structure consists in a 2D hexagonal DNA assembly embedded inside the water channels of a lamellar phase of lipid bilayers.

As the stacking period of the lamellar phase $D$, deduced from the equation $D=a \sqrt{3} / 2$, is $D=5.35 \mathrm{~nm}$, the corresponding water channel thickness evaluates to $D-\delta=$ $1.75 \mathrm{~nm}$, i.e. a value less than the DNA diameter. In this

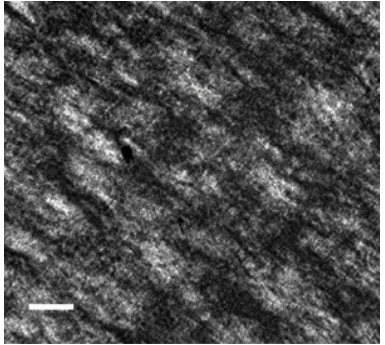

Fig. 9: Lamellar texture of the dried supported film as observed with polarised light microscopy. Scale bar: $10 \mu \mathrm{m}$.

strongly confined DNA-lipid complex, the lipid bilayers are thus distorted, an effect already observed in a similar context [28].

Discussion and conclusion. - We describe a new structural organisation in a highly dehydrated DNAlipid complex, where DNA rods form a $2 \mathrm{D}$ hexagonal structure that intercalates in between the lipid bilayers of a lamellar structure. Though other two-dimensionally ordered DNA-lipid hydrated complexes have previously been found, where either the lamellar lipid structure was associated to a centred-rectangular DNA assembly, or both lipids and DNA displayed hexagonal order, such a 2D hexagonal (DNA)-lamellar (lipids) structure is here observed for the first time. In contrast with other studies where the DNA-lipid complexes are prepared in excess water, we control the amount of water present in the system, and dehydrate the complex significantly below its swelling limit. This amounts to varying the confinement of the DNA molecules by the lipid bilayers, acting as soft "walls". The replacement of the dilute DNA-lipid, possibly $2 \mathrm{D}$ nematic, complex by one with the $2 \mathrm{D}$ hexagonal symmetry, though still displaying the lamellar structure for the lipid component may thus be seen as a confinementinduced structural transition.

It is important to note that the confining lipid "walls" are truly soft: we have checked, using wide-angle X-ray scattering on powder samples with similar compositions, that the lipids are self-assembled in liquid $\left(L_{\alpha}\right)$ bilayers, as indicated by the presence of the broad line at ca. $15 \mathrm{~nm}^{-1}$ (data not shown), characteristic of the disordered state of the lipid hydrophobic tails [41].

Our system appears to be original and well suited to the study of confinement effects in DNA-lipid, and possibly other, hydrated complexes. This opens new perspectives in the quest for the theoretically described "sliding phase", with a kind of order intermediate between the 2D nematic within lamellar and 2D crystalline within lamellar orders [38]. The detailed mechanism of the DNA-lipid complex formation remains somehow elusive, however, as electrostatic complexation is not the obvious candidate, and more theory, extending to concentrated phases the Flory approach discussed in ref. [35] for instance, would be desirable. Still, the experimental constraints of 
grazing incidence small-angle scattering turn into advantages as far as sample orientation and hydration are concerned. Similar humidity control devices may be used for complementing the present structural approach by studying the phase transitions with optical microscopy, as well as the dynamic properties of the DNA rods in oriented samples with various levels of confinement using fluorescence recovery after photo-bleaching. The latter investigations are currently under progress.

$$
* * *
$$

We thank the European Synchrotron Radiation Facility for allocating the beam time and our local contact J.-S. MichA for his invaluable help in setting the experiment. EADO and ERTDS thank Conselho Nacional de Desenvolvimento Científico e Tecnológico (CNPq), as well as Fundação de Amparo à Pesquisa do Estado de São Paulo (FAPESP) for grants. This work received support from the bi-national Brazilian-French program CAPES/CoFÉCUB, project Ph558/07.

\section{REFERENCES}

[1] Yамамото J. and Tanaka H., Nat. Mater., 4 (2005) 75.

[2] Angelatos A. S., Katagiri K. and Caruso F., Soft Matter, 2 (2006) 18.

[3] Zhang Z. and van Duijneveldt J. S., Soft Matter, 3 (2007) 596.

[4] Binder K., Horbach J., Vink R. and DE Virgillis A., Soft Matter, 4 (2008) 1555.

[5] Dutta D., Pujari P. K., Sudarshan K. and Sharma S. K., J. Phys. Chem. C, 112 (2008) 19055.

[6] Nygård K., Satapathy D. K., Buitenhuis J., Perret E., Bunk O., David C. and van der Veen J. F., EPL, 86 (2009) 66001.

[7] Binder K., Landau D. P. and Ferrenberg A. M., Phys. Rev. Lett., 74 (1995) 298.

[8] Gelb L. D., Gubbins K. E., Radhakrishnan R. and Silwinska-Bartkowiak M., Rep. Prog. Phys., 62 (1999) 1573.

[9] Odriozola G., Jiménez-Ángeles F. and LozadaCassou M., Phys. Rev. Lett., 97 (2006) 018102.

[10] Sha M., Wu G., LiU Y., Tang Z. and FAng H., J. Phys. Chem. C, 113 (2009) 4618.

[11] Pusey P. N. and van Megen W., Nature, 320 (1986) 340.

[12] Pusey P. N., van Megen W., Bartlett P., Ackerson B. J., Rarity J. G. and Underwood S. M., Phys. Rev. Lett., 63 (1989) 2753.

[13] Kékicheff P., Cabane B. and Rawiso M., J. Colloid Interface Sci., 102 (1984) 51.

[14] Fabre P., Casagrande C., Veyssié M., Cabuil V. and Massart R., Phys. Rev. Lett., 64 (1990) 539.

[15] Ott A., Urbach W., Langevin D., Ober R. and Waks M., Europhys. Lett., 12 (1990) 395.
[16] Arrault J., Grand C., Poon W. C. K. and Cates M. E., Europhys. Lett., 38 (1997) 625.

[17] RÄdler J. O., Koltover I., SAlditt T. and SAfinya C. R., Science, 275 (1997) 810.

[18] Yang P., Zhao D., Margolese D. I., Chmelka B. F. and Stucky G. D., Nature, 396 (1998) 152.

[19] Béneau K., Constantin D., Davidson P., Dessombz A. and ChanéaC C., Langmuir, 24 (2008) 8205.

[20] Dif A., Henry E., Artzner F., Baudy-Floc'h M., Schmultz M., Dahan M. and Marchi-Artzner V., J. Am. Chem. Soc., 130 (2008) 8289.

[21] Kamperman M., Fierke M. A., Garcia C. B. W. and WiESNER U., Macromolecules, 41 (2008) 8745.

[22] Angelov B., Angelova A., Vainio U., Garamus V. M., Lesieur S., Willumeit R. and Couvreur P., Langmuir, 25 (2009) 3734.

[23] Koyonova R. and Tenchov B., Soft Matter, 5 (2009) 3187.

[24] Madhusudhana Rao N. and Gopal V., Biosci. Rep., 26 (2006) 301.

[25] Felgner P., Gadek T. R., Holm M., Roman R., Chan H. W., Wenz M., Northrop J. P., Ringold G. M. and Dnielsen M., Proc. Natl. Acad. Sci. U.S.A., 84 (1987) 7413.

[26] Tarahovsky Y. S., Khusainova R. S., Gorelov A. V., Niclaeva T. I., Deev A. A., Dawson A. K. and Ivanitsky G. R., FEBS Lett., 390 (1996) 133.

[27] Potт T. and Roux D., FEBS Lett., 511 (2002) 150.

[28] Mcmanus J. J., Rädler J. O. and Dawson K. A., Langmuir, 19 (2003) 9630.

[29] Pott T., Colin A., Navailles L. and Roux D., Interface Sci., 11 (2003) 249.

[30] Mcmanus J. J., RÄdler J.O. and Dawson K. A., J. Am. Chem. Soc., 126 (2004) 15966.

[31] Koyonova R. and MacDonald R. C., Nano Lett., 4 (2004) 1475.

[32] Hohner A., Bayer J. and Rädler J. O., Eur. Phys. J. E, 21 (2006) 41.

[33] Uhríková D., Lengyel A., Hanulová M., Funari S. S. and Balgavý P., Eur. Biophys. J., 375 (2007) 363.

[34] Caracciolo G., Pozzi D., Caminiti R., Mancini G., Luciani P. and Amenitsch H., J. Am. Chem. Soc., 129 (2007) 10092.

[35] Colin A. and Roux D., Eur. Phys. J. E, 8 (2002) 499.

[36] Koltover I., Salditt T., RÄdler J. O. and SAfinya C. R., Science, 281 (1998) 78.

[37] Artzner F., Zantl R., Rapp G. and Rädler J. O., Phys. Rev. Lett., 81 (1998) 5015.

[38] Golubović L., Lubensky T. C. and O'Hern C. S., Phys. Rev. E, 62 (2000) 1069.

[39] Pieranski P., J. Phys.: Condens. Matter, 17 (2005) s3333.

[40] Oswald P. and Pieranski P., Smectic and Columnar Liquid Crystals. Concepts and Physical Properties Illustrated by Experiments (CRC Press) 2005.

[41] Ranck J. L., Keira T. and Luzzati V., Biochim. Biophys. Acta, 488 (1977) 432. 